03,11

\title{
Пути политипных превращений в карбиде кремния
}

\author{
(C) C.A. Кукушкин ${ }^{1,2,3}$, А.В. Осипов ${ }^{2}$ \\ ${ }^{1}$ Институт проблем машиноведения РАН, \\ Санкт-Петербург, Россия \\ ${ }^{2}$ Санкт-Петербургский национальный исследовательский университет \\ информационных технологий, механики и оптики, \\ Санкт-Петербург, Россия \\ ${ }^{3}$ Санкт-Петербургский государственный политехнический университет Петра Великого, \\ Санкт-Петербург, Россия \\ E-mail: sergey.a.kukushkin@gmail.com
}

Поступила в Редакцию 10 апреля 2019 г.

В окончательной редакции 11 апреля 2019 г.

Принята к публикации 11 апреля 2019 г.

\begin{abstract}
Методами $a b$ initio изучены два основных политипных превращения в карбиде кремния, а именно, $2 H \rightarrow 6 H$ и $3 C \rightarrow 6 H$. Показано, что промежуточные фазы с тригональной симметрией $P 3 m 1$ и моноклинной симметрией $\mathrm{Cm}$ сильно облегчают перемещения плотноупакованных слоев при таких переходах, разбивая их на отдельные стадии. Обнаружено, что два данных политипных превращения протекают совершенно по-разному. При переходе $2 H \rightarrow 6 H$ перемещаемые связи заметно наклоняются по сравнению с исходным положением, что позволяет уменьшить сжатие связей $\mathrm{SiC}$ в плоскости (112̄0). Переход $3 \mathrm{C} \rightarrow 6 H$ осуществляется посредством образования вспомогательных связей $\mathrm{Si}-\mathrm{Si}$ и $\mathrm{C}-\mathrm{C}$, живущих короткое время и помогающих плотноупакованным слоям поменяться местами. В результате активационный барьер превращения $2 \mathrm{H} \rightarrow 6 \mathrm{H}$ (1.7 eV/atom) существенно меньше активационного барьера превращения $3 \mathrm{C} \rightarrow 6 \mathrm{H}$ (3.6 eV/atom), что означает, что второй переход должен протекать при температурах на $750-800^{\circ} \mathrm{C}$ выше, чем первый. Рассчитаны энергетические профили данных политипных превращений, а также геометрии всех промежуточных и переходных фаз. Показано, что все переходные состояния имеют моноклинную симметрию.
\end{abstract}

Ключевые слова: фазовые переходы, политипы, карбид кремния, метод упругих лент, метод функционала плотности.

DOI: $10.21883 /$ FTT.2019.08.47967.452

\section{1. Введение}

Хорошо известно, что плотноупакованные слоистые кристаллические соединения, такие как карбид кремния $\mathrm{SiC}$, сульфид цинка $\mathrm{ZnS}$, иодид кадмия $\mathrm{CdI}_{2}$, сульфид молибдена $\mathrm{MoS}_{2}$ обладают большим количеством фаз, отличающихся друг от друга порядком чередования атомных слоев [1]. Например, $\mathrm{SiC}$ обладает более чем 100 политипами с примерно одинаковой энергией на атом [2]. Свойства политипов могут значительно отличаться друг от друга, что может быть использовано для работы различных приборов [3]. В частности, ширина запрещенной зоны кубического политипа $\mathrm{SiC}-3 \mathrm{C}$ равна $2.4 \mathrm{eV}$, гексагонального политипа $\mathrm{SiC}-4 H-3.3 \mathrm{eV}$, причем подвижность электронов в этих политипах очень высокая, поэтому приборы на основе гетероперехода $3 C / 4 H$ считаются очень перспективными [3]. С этой точки зрения важно понять, каким образом один политип превращается в другой.

Поскольку политипы соответствуют минимумам на поверхности энергии Гиббса, то при определенных условиях возможны как прямые, так и обратные переходы из одного политипа в другой. Например, при повышении температуры в кристаллах $\mathrm{SiC}-2 H$ наблюдаются переходы $2 H \rightarrow 6 H$ [4], причем самые первые признаки перехода появляются уже при $400^{\circ} \mathrm{C}$. Обратные переходы
$6 H \rightarrow 2 H$ наблюдаются при высоком давлении и высокой концентрации бора или алюминия $[1,5]$, которые, очевидно, делают политип $2 H$ более выгодным, а политип $6 H$ - менее выгодным. Превращения неоднократно наблюдались при температурах $1600-2100^{\circ}$ [1,5-7]. Обратные переходы $6 H \rightarrow 3 C$ наблюдаются при температуре порядка $1200-1400^{\circ} \mathrm{C}$ и давлении $30-70 \mathrm{kbar}$ [8].

Для описания переходов из одного политипа в другой было предложено несколько моделей $[1,5,9]$ как чисто твердофазных, так и с использованием газообразной или жидкой фазы. Однако, в карбиде кремния, как показывают экспериментальные данные $[1,10]$, реализуется в основном твердофазный механизм смещения слоев (layer displacement fault). В настоящей работе методами квантовой химии исследуются два наиболее актуальных, но совершенно разных по своему характеру превращения политипов карбида кремния, а именно, $2 \mathrm{H} \rightarrow 6 \mathrm{H}$ и $3 C \rightarrow 6 H$. При переходе $2 H \rightarrow 6 H 2$ слоя из каждых 6 должны изменить свое положение в кристалле

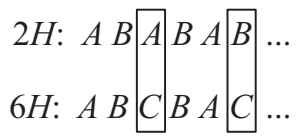

Здесь буквами $A, B$ и $C$ обозначены все три возможных положения плотноупакованных слоев $\mathrm{SiC}$ [1]. Один слой 
атомов в положении $A$ должен переместиться в положение $C$, и один слой атомов в положении $B$ также должен переместиться в положение $C$ (эти слои выделены прямоугольником). При переходе $3 C \rightarrow 6 H 2$ соседних слоя из каждых 6 должны поменяться местами:

$$
\begin{aligned}
& 3 C: A B C A B C \ldots \\
& 6 H: A B C B A C \ldots
\end{aligned}
$$

Слой атомов в положении $A$ должен перейти в положение $B$, а соседний с ним слой $B$, наоборот, должен переместиться в положение $A$ (эти слои выделены прямоугольником). Второй случай, очевидно, должен реализовываться значительно сложнее первого, поскольку, когда два соседних слоя меняются местами, некоторые атомы мешают перемещаться друг другу.

В настоящей работе оба превращения исследуются методами $a b$ initio, что позволяет детально проанализировать механизм перемещения слоев и сравнить их между собой.

\section{2. Постановка задачи и методы исследования}

В обоих случаях для описания процесса перемещения слоев атомов $\mathrm{SiC}$ достаточно рассмотреть всего одну ячейку кристаллической решетки $\mathrm{SiC}-6 \mathrm{H}$ (или три ячейки $\mathrm{SiC}-2 H$ по оси $z$ и две ячейки $\mathrm{SiC}-3 C$ в проекции (111)), т.е. всего 12 атомов (6 атомов $\mathrm{Si}$ и 6 атомов С). Для простоты будем считать размеры рассматриваемой системы в процессе перехода неизменными и равными размерам ячейки $\mathrm{SiC}-6 H$, а именно, $a=b=3.08 \AA, c=15.12 \AA$. По всем трем осям накладываются периодические граничные условия.

На рис. 1, $a$ исходная система из 3 ячеек $\mathrm{SiC}-2 \mathrm{H}$ изображена слева, а конечная система из 1 ячейки $\mathrm{SiC}-6 H$ - справа на рис. $1, b$. Для наглядности показаны все 28 атомов, принадлежащие кристаллическим ячейкам, а не только 12 независимых. Вертикальная связь $\mathrm{Si}-\mathrm{C}$, соответствующая плотноупакованному слою $\mathrm{SiC}$, перемещается из положения $A$ в положение $C$ (см. pис. $1, a)$ и из положения $B$ в положение $C$ (рис. $1, b)$. Исходная система из 2 ячеек $\mathrm{SiC}-3 C$ изображена слева на рис. 2, а конечная система из 1 ячейки $\mathrm{SiC}-6 \mathrm{H}-$ справа на рис. 2. Соседние связи в положениях $A$ и $B$ меняются местами (рис. 2).

В квантовой химии подобные перемещения атомных слоев лучше всего описываются методом упругих лент (Nudged Elastic Band (NEB)) [11,12]. Идея данного метода заключается в том, что, зная начальное и конечное положения атомов, можно заморозить систему и ввести в неё дополнительные силы, которые направят атомы от начального состояния к конечному. При выполнении определенных условий система пойдёт по пути с минимальным перепадом энергии (Minimal Energy Pathway (MEP)) [12]. Это такой путь превращения, что
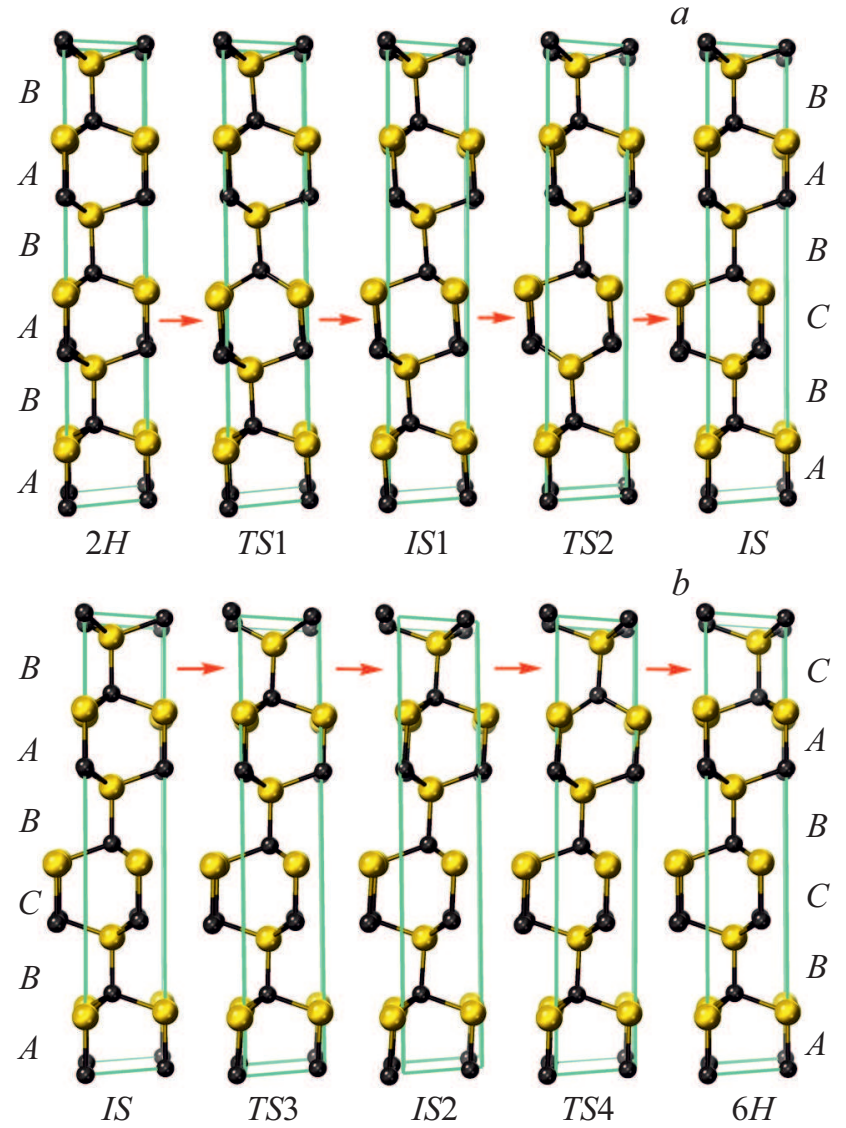

Рис. 1. Превращение политипа $\mathrm{SiC}-2 H$ в политип $\mathrm{SiC}-6 H$ путем перемещения в другое положение по очереди двух независимых плотноупакованных слоев, рассчитанное методами $a b$ initio. Большие светлые шарики - атомы $\mathrm{Si}$, маленькие темные - атомы С. Начальную $2 H$ и конечную фазу $6 H$ перехода разделяет метастабильная промежуточная фаза $I S$ с тригональной симметрией, сильно облегчающая данное превращение. Часть $a$ показывает первую половину превращения, т. е. из $2 H$ в $I S$, часть $b-$ вторую половину, т.е. из $I S$ в $6 H$. Седловым точкам с наибольшей энергией отвечают переходные фазы $T S 1, T S 2$ и $T S 3$.

любое локальное изменение пути приводит к увеличению энергии системы в окрестности данной точки пути. Иными словами, это путь наискорейшего спуска из исходной конфигурации в конечную конфигурацию, то есть наиболее вероятный путь перехода. В теории фазовых переходов первого рода $[13,14]$ координата вдоль такого пути из исходной фазы в конечную является параметром порядка данного фазового перехода. Соответственно, сечение поверхности потенциальной энергии вдоль пути превращения является энергетическим профилем данного превращения. Несомненным достоинством метода упругих лент является то, что все промежуточные и переходные фазы могут быть найдены, если известны лишь начальная и конечная конфигурация системы [12].

Энергия системы в каждой точке пути (энергия так называемых изображений системы [12]) в данной работе вычислялась методом функционала плотности $[15,16]$ 


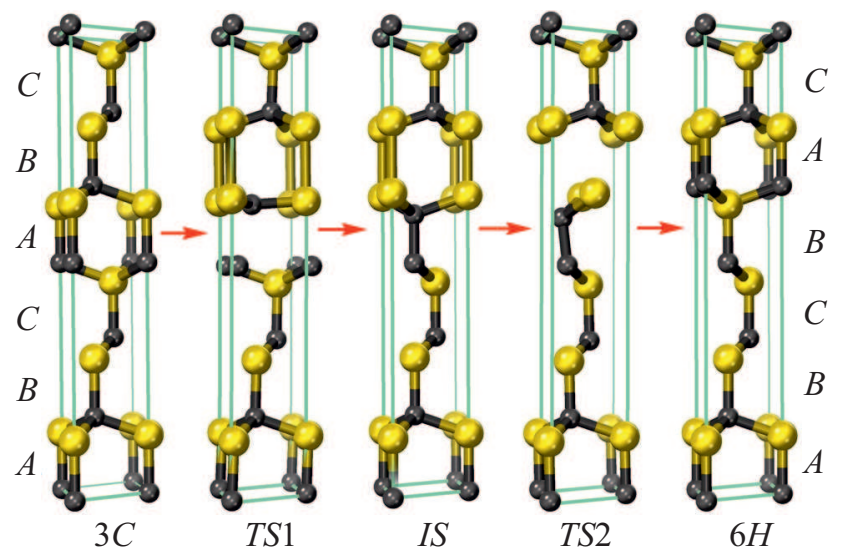

Рис. 2. Превращение политипа $\mathrm{SiC}-3 C$ в политип $\mathrm{SiC}-6 H$ путем обмена положениями двух соседних плотноупакованных слоев, рассчитанное методами $a b$ initio. Большие светлые шарики - атомы $\mathrm{Si}$, маленькие темные - атомы С. Начальную $3 C$ и конечную фазу $6 H$ перехода разделяет метастабильная промежуточная фаза $I S$ с моноклинной симметрией, сильно облегчающая данное превращение. Седловым точкам с наибольшей энергией отвечают переходные фазы TS1 и $T S 2$.

в базисе плоских волн. Для моделирования использовался пакет Medea-Vasp, использующий псевдопотенциалы проекционных присоединенных волн (Projector Augmented Waves, PAW) [17]. Обменно-корреляционная энергия рассчитывалась в рамках функционала PBE [18], соответствующему обобщенно-градиентному приближению (Generalized Gradient Approximation, GGA). Энергия обрезания плоских волн во всех расчетах была равна $400 \mathrm{eV}$. В обратном пространстве вычисления проводились по сетке Монхорста-Пака из $7 \times 7 \times 3$ точек, отвечающей расстоянию между точками $0.31 / \AA$.

\section{3. Обсуждение результатов и выводы}

Применение метода NEB к микроскопическому описанию переходов между политипами карбида кремния с использованием метода функционала плотности выявило наличие нескольких четко выраженных промежуточных минимумов в зависимости энергии системы от пути превращения МЕР. При переходе $2 H \rightarrow 6 H$ таких минимумов три (рис. 3). Существование центрального минимума (промежуточного состояния $I S$ ) связано с тем, что две связи, которые меняют свои положения, делают это не одновременно, а по очереди. В частности, на рис. $1, a$ и $1, b$ изображена ситуация, когда вначале слой $A$ переходит в $C$, лишь затем $B$ переходит в $C$. Этот переход иллюстрируется схемой

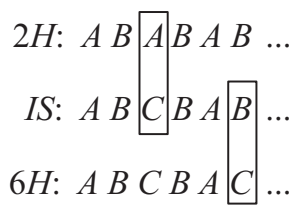

Кроме этого перехода возможен переход, когда вначале слой $B$ переходит в $C$, и лишь затем слой $A$ переходит в $C$ :

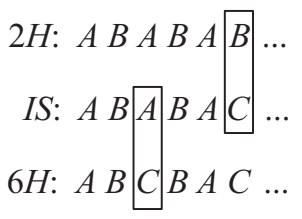

Расчеты показывают, что с энергетической точки зрения оба варианта почти тождественны, но первый вариант все же чуть предпочтительнее. Подчеркнем, что в обоих случаях промежуточное состояние $I S$ имеет симметрию $P 3 m 1$, что ниже чем исходная и конечная симметрии $P 6_{3} m c$. Параметры решетки фазы IS совпадают с параметрами решетки конечной фазы. Интересно отметить, что в первом случае структура промежуточного состояния IS (ABCBABA..) очень похожа на политип $4 H$, поскольку первые 6 слоев $I S$ совпадают с $4 H(A B C B A B C \ldots)$, отличия начинаются лишь с седьмого слоя.

Расчеты методом NEB показывают, что переходы $2 H \rightarrow I S$ и $I S \rightarrow 6 H$ также содержат по одному промежуточному состоянию $I S 1$ (рис. $1, a)$ и $I S 2$ (рис. $1, b$ ) соответственно. Эти промежуточные метастабильные состояния имеют еще меньшую симметрию $\mathrm{Cm}$. Примитивная ячейка фаз IS1 и IS2 содержит 6 атомов $\mathrm{Si}$ и 6 атомов $\mathrm{C}$ и имеет параметры $a=b=3.08 \AA$, $x=16.03 \AA, \alpha=\beta=106.75^{\circ}, \gamma=60^{\circ}$. Промежуточные фазы IS1 и IS2 отделены от исходной фазы $2 H$, промежуточной фазы $I S$ и конечной фазы $6 H$ тремя максимумами, то есть переходными фазами $T S 1, T S 2$, $T S 3$ и TS4 соответственно (см. рис. 3). Симметрия примитивных ячеек $T S 1, T S 2, T S 3$ и $T S 4$ совпадают с симметрией ячеек $I S 1$ и $I S 2$. Параметры решеток примитивных ячеек $T S 1, T S 2, T S 3$ и $T S 4$ оказались равны

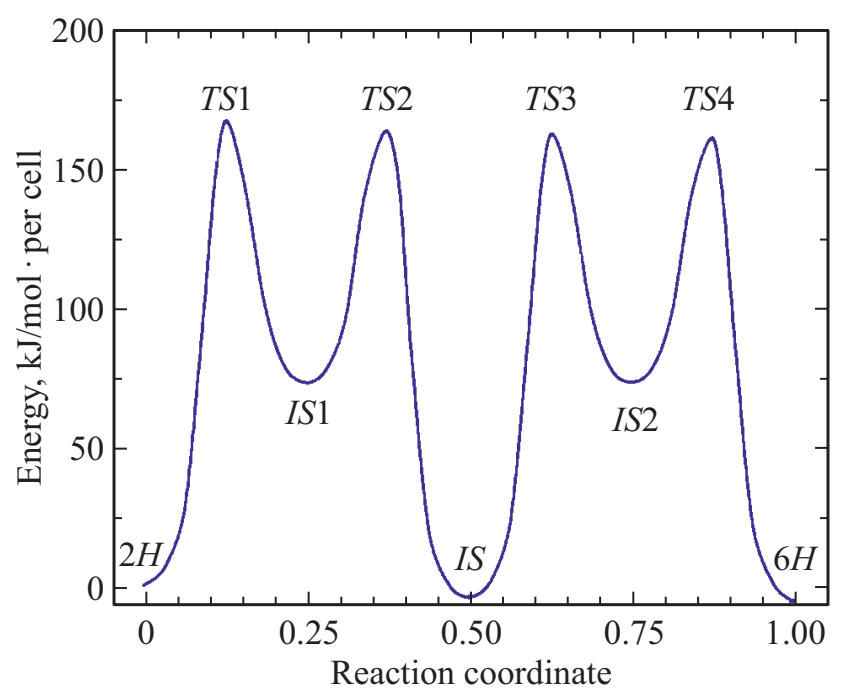

Рис. 3. Энергетический профиль превращения политипа $\mathrm{SiC}-2 H$ в политип $\mathrm{SiC}-6 H$, рассчитанный методом упругих лент NEB $[11,12]$. 
$a=b=3.08 \AA, c=16.03 \AA, \alpha=\beta=106.75^{\circ}, \gamma=60^{\circ}$, т. е. они совпадают с $I S 1$ и $I S 2$. Энергетический профиль всего превращения $2 H \rightarrow 6 H$ изображен на рис. 3 . Высота активационного барьера перемещения одного плотноупакованного слоя совпадает с разницей энергий состояний $T S 3$ и $I S$ и равна $165 \mathrm{~kJ} / \mathrm{mol}(1.7 \mathrm{eV} /$ atom). Если бы промежуточных состояний $I S 1$ и IS2 не существовало, то высота активационного барьера перехода $2 H \rightarrow 6 H$ была бы значительно больше и равна примерно $3.0 \mathrm{eV} /$ atom. Это связано с тем, что при „прямом“ переходе приходится очень сильно сжимать связи перемещаемых атомов с ближайшими неперемещаемыми атомами. Поэтому атомы $\mathrm{Si}$ и $\mathrm{C}$ используют другой, более выгодный путь перехода с участием промежуточных фаз IS1 и IS2. Первым начинает перемещаться атом $\mathrm{Si}$ (рис. 1), при этом вертикальная связь $\mathrm{Si}-\mathrm{C}$ наклоняется. Наклон перемещаемых связей позволяет уменьшить сжатие соседних связей в плоскости $(11 \overline{2} 0)$ почти в 2 раза. Затем основное перемещение совершает атом C (рис. 1). Анализ собственных частот состояний TS1, $T S 2, T S 3$ и $T S 4$ показал, что помимо положительных собственных частот переходные фазы $T S 1, T S 2, T S 3$ и TS4 имеют единственную отрицательную (мнимую) частоту, которая лежит в интервале $400-415 \mathrm{~cm}^{-1}$. Данный факт доказывает, что фазы $T S 1, T S 2, T S 3$ и $T S 4$ являются переходными и соответствуют седловым точкам поверхности энергии Гиббса [19].

Подчеркнем, что сходимость итераций в методе упругих лент NEB для данного превращения очень хорошая. При этом она существенно улучшается, если в качестве начального приближения выбрать кривую, проходящую через состояние $I S$. В этом случае энергетический профиль перехода $2 H \rightarrow 6 H$ (рис. 3) вычисляется методом NEB c 25 изображениями при сходимости с точностью $0.1 \mathrm{eV} / \AA$ за 70-100 итераций. В противном случае требуется более 200 итераций при точности $0.2 \mathrm{eV} / \AA$.

Переход $3 C \rightarrow 6 H$ описывается методом NEB несопоставимо сложнее. Начальное приближение в виде линейной зависимости между исходной и конечной конфигурацией вообще не сходится к решению из-за того, что, когда соседние слои пытаются поменяться местами, некоторые атомы мешают друг другу. В результате метод упругих лент самостоятельно не может найти решение. Поэтому, путем оптимизации геометрии систем, близких к промежуточному, вначале находится промежуточная фаза $I S$, изображенная в середине на pис. 2 , и лишь затем в качестве начального приближения выбирается путь, проходящий через начальное состояние $3 C$, промежуточное $I S$ и конечное $6 H$. Даже в этом случае сходимость итераций не очень хорошая, но за $100-150$ итераций при 25 изображениях системы можно добиться сходимости с точностью $0.5 \mathrm{eV} / \AA$ А. Результаты расчетов показаны на рис. 2 и 4.

Ключевым результатом данных расчетов является то, что для осуществления перехода $3 C \rightarrow 6 H$ требуется разрыв $\mathrm{Si}-\mathrm{C}$ связей, образование вместо них новых

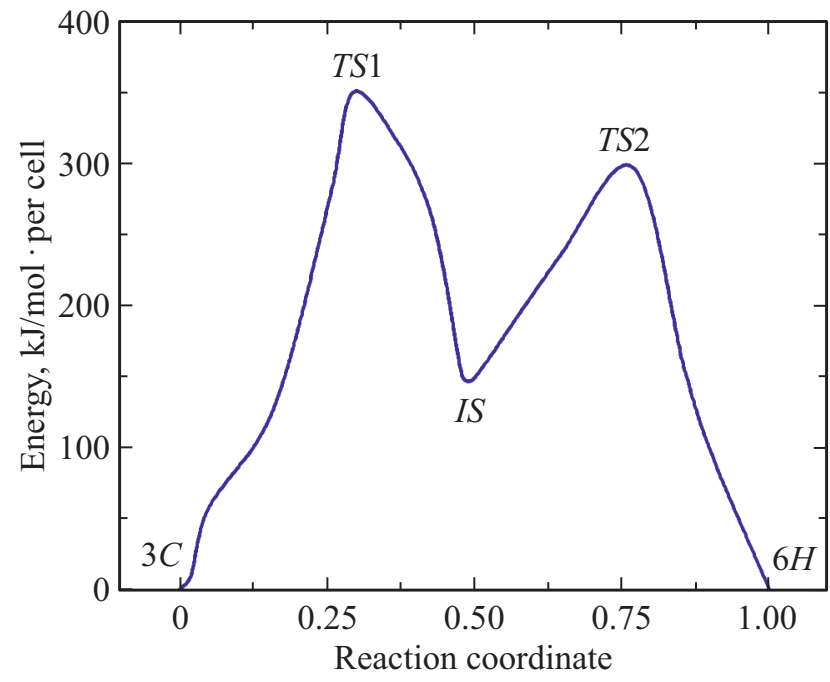

Рис. 4. Энергетический профиль превращения политипа $\mathrm{SiC}-3 C$ в политип $\mathrm{SiC}-6 H$, рассчитанный методом упругих лент NEB $[11,12]$.

$\mathrm{Si}-\mathrm{Si}$ и $\mathrm{C}-\mathrm{C}$ связей, затем их разрыв, и лишь затем образование новых $\mathrm{Si}-\mathrm{C}$ связей (см. рис. 2). Образование $\mathrm{Si}-\mathrm{Si}$ и $\mathrm{C}-\mathrm{C}$ связей в $\mathrm{SiC}$ требует больших затрат энергий, поэтому высота барьера данного перехода заметно больше высоты перехода $2 H \rightarrow 6 H$ и равна $350 \mathrm{~kJ} / \mathrm{mol}$ (3.6 eV/atom) (см. рис. 4). Если начало политипного превращения $2 \mathrm{H} \rightarrow 6 \mathrm{H}$ примерно соответствует $400^{\circ} \mathrm{C}$, то политипное превращение $3 \mathrm{C} \rightarrow 6 \mathrm{H}$ начинается примерно при $1150^{\circ} \mathrm{C}$.

Фаза $I S$ для перехода $3 C \rightarrow 6 H$ обладает тригональной симметрией $P 3 m 1$, что ниже чем симметрия исходной фазы $F \overline{4} 3 m$ и симметрия конечной фазы $P 6_{3} m c$. Параметры решетки фазы $I S$ совпадают с параметрами решетки конечной фазы. Примитивные ячейки переходных фаз $T S 1$ и $T S 2$ обладают симметрией $C m$ и имеют параметры $a=b=3.08 \AA, c=16.03 \AA, \alpha=\beta=106.75^{\circ}$, $\gamma=60^{\circ}$. Анализ собственных частот состояний TS1 и TS2 показал, что помимо положительных собственных частот переходные фазы TS1 и TS2 имеют единственную отрицательную (мнимую) частоту, а именно, -460 и $-230 \mathrm{~cm}^{-1}$, соответственно. Данный факт доказывает, что фазы TS1 и TS2 являются переходными и соответствуют седловым точкам поверхности энергии Гиббса [19].

Таким образом, моделирование политипных превращений в карбиде кремния методами квантовой химии показало, что если один плотноупакованный слой меняет свое положение, то такой переход протекает достаточно просто путем наклона перемещаемых связей. Высота активационного барьера такого перехода равна $165 \mathrm{~kJ} / \mathrm{mol}(1.7 \mathrm{eV} /$ atom $)$, что соответствует температуре начала перехода $400^{\circ} \mathrm{C}$. Если же несколько соседних плотноупакованных слоев одновременно меняют свое положение, например, меняются местами, то в этом случае переход протекает гораздо сложнее через образо- 
вание вспомогательных $\mathrm{Si}-\mathrm{Si}$ и $\mathrm{C}-\mathrm{C}$ связей. В этом случае высота активационного барьера значительно выше, например, при переходе $3 C \rightarrow 6 H$ она равна $350 \mathrm{~kJ} / \mathrm{mol}$ (3.6 eV/atom), что соответствует температуре начала перехода $1150^{\circ} \mathrm{C}$. Обе рассчитанные температуры неплохо согласуются с экспериментальными данными по данным политипным превращениям [1,4-8]. Во всех случаях промежуточные состояния играют очень важную роль в политипных превращениях, поскольку значительно снижают активационный барьер превращения. Более симметричные промежуточные метастабильные фазы в карбиде кремния имеют тригональную симметрию $P 3 m 1$, менее симметричные - моноклинную симметрию $\mathrm{Cm}$. Bce переходные состояния также имеют моноклинную симметрию $\mathrm{Cm}$.

\section{Финансирование работы}

Работа выполнена при финансовой поддержке РФФИ (грант № 16-29-03149-офи-м).

Работа выполнена при использовании оборудования Уникального стенда (УНО) „Физика, химия и механика кристаллов и тонких пленок“ ФГУП ИПМаш РАН.

\section{Конфликт интересов}

Авторы заявляют, что у них нет конфликта интересов.

\section{Список литературы}

[1] M.T. Sebastian, P. Krishna. Random, non-random and periodic faulting in crystals. Routledge, Taylor \& Francis Group, London and N.Y. (2014). 383 p.

[2] J. Fan, P.K. Chu. Silicon Carbide Nanostructures. Fabrication, Structure, and Properties. Springer, (2014). 330 p.

[3] P.V. Bulat, A.A. Lebedev, Yu.N. Makarov. Sci. Techn. J. Inform. Technol., Mech. Opt. 3, (91), (2014); (https://openbooks.ifmo.ru/read_ntv/9619/9619.pdf)

[4] T. Tagai, S. Sueno, R. Sadanaga. Mineralog. J. 6, 340 (1971).

[5] N.W. Jepps, T.F. Page. Prog. Cryst. Growth Charact. 7, 259 (1983).

[6] H.N. Baumann. J. Electrochem. Soc. 99, 109 (1952).

[7] S.I. Vlaskina, G.N. Mishinova, V.I. Vlaskin, V.E. Rodionov, G.S. Svechnikov. Semicond. Phys., Quantum Electron. \& Optoelectron. 14, 432 (2011).

[8] М.И. Сохор, В.Г. Кондаков, Л.И. Фельдгун. Докл. АН CCCP, 175, 4, 826 (1967).

[9] А.А. Лебедев, С.Ю. Давыдов, Л.М. Сорокин, Л.В. Шахов. Письма в ЖТФ 41, 23, 89 (2015).

[10] D. Pandey, S. Lele, P. Krishna. Proc. Roy. Soc. Lond. A 369, 463 (1980).

[11] G. Henkelman, B.P. Uberuaga, H. Jonsson. J. Chem. Phys. 113, 9901 (2000).

[12] K.J. Caspersen, E.A. Carter. PNAS 102, 6738 (2005).

[13] A.V. Osipov. J. Phys. D: 28, 1670 (1995).

[14] A.V. Osipov. Thin Solid Films. 261, 173 (1995).

[15] J.G. Lee. Computational Materials Science. An introduction. CRS Press, Taylor \& Francis Group, Roca Baton (2017). $351 \mathrm{p}$.
[16] D.S. Sholl, J.A. Steckel. Density functional theory. A practical introduction. J. Wiley \& Sons, Hoboken (2009). 238 p.

[17] J. Hafner. J. Comp. Chem. 29, 2044 (2008).

[18] J.P. Perdew, K. Burke, M. Ernzerhof. Phys. Rev. Lett. 77, 3865 (1996).

[19] P. Atkins, J. de Paula. Atkins' Physical Chemistry. Univ. Press, Oxford (2006). 1067 p.

Редактор Ю.Э. Китаев 